\title{
Revisión de Caso Clínico: \\ Goma o absceso tuberculoso metastásico como diagnóstico inicial de tuberculosis en un paciente inmunocompetente: una presentación inusual
}

\author{
A Marco, R Solé ${ }^{1}$ E Raguer ${ }^{1}$, M Aranda ${ }^{1}$
}

Servicios Sanitarios del Centro Penitenciario de Hombres de Barcelona (CPHB) y

Servicio de Medicina Interna del Hospital Consorci Sanitari de Terrasa (HCST) ${ }^{1}$.

\section{RESUMEN}

Fundamento y Objetivo: Los abscesos fríos tuberculosos o gomas son inusuales y producto de la diseminación hematógena de micobacterias latentes, formando abscesos en extremidades o tronco, generalmente sin compromiso de tejidos profundos. Se presenta un caso de goma como diagnóstico inicial de tuberculosis diseminada.

Métodos: Caso estudiado en 2012 por los servicios médicos del centro penitenciario de Hombres de Barcelona y el Servicio de Medicina Interna del Hospital Consorci Sanitari de Terrassa. Se recogen los datos de la historia clínica, de los informes hospitalarios y de las pruebas complementarias practicadas.

Resultados: Preso, 35 años, de Cabo Verde, residente en España desde los 4 años. IDR Mantoux positiva. Inmunocompetente. Por síndrome constitucional se programó estudio hospitalario. Exploración anodina, excepto palpación de masa en región lumbar. La punción aspirativa fue positiva para M. tuberculosis mediante PCR y cultivo Lowenstein. La TC mostró nódulos pulmonares, apical D cavitado y en LSD. Tras aislamiento respiratorio, tratamiento antituberculoso y excelente evolución fue dado de alta con diagnóstico de tuberculosis diseminada.

Discusión: Se recomienda vigilar lesiones que, aunque infrecuentes, pueden ser secundarias a infección por $M$ tuberculosis, especialmente en población inmunocomprometida y en inmigrantes procedentes de zonas hiperendémicas de tuberculosis.

Palabras clave: Prisiones; Tuberculosis cutánea; España; Diagnóstico diferencial; Enfermedades Endémicas; Absceso; VIH.

\section{TUBERCULOUS GUMMA OR METASTATIC TUBERCULOUS ABSCESS AS INITIAL DIAGNOSIS OF TUBERCULOSIS IN AN IMMUNOCOMPETENT PATIENT: AN UNUSUAL PRESENTATION}

\begin{abstract}
Background and Objectives: Tuberculous cold abscesses or gumma are an unusual form of tuberculosis. We report a case of gumma as initial diagnosis of disseminated tuberculosis.

Method: This case was studied in 2012 in Barcelona (Spain). Source data was compiled from the electronic clinical records, hospital reports and additional diagnostic testing.

Results: Immunocompetent inmate, born in Cape Verde, living in Spain since the age of four. Positive tuberculin skin test. Initial examination without interest, but a palpable mass in lower back. Fine needle aspiration of the abscess was positive (PCR and Lowenstein) for $M$. tuberculosis. Computed tomography showed lung cavitary nodes in apical part and lung upper right side. After respiratory isolation, antituberculous therapy and an excellent evolution, the patient was discharged from hospital with disseminated tuberculosis diagnosis.

Discussion: It is advisable to monitor the injuries since, although rare, it may be secondary to Mycobacterium tuberculosis infection, mainly in inmuno-compromised populations and in immigrants coming from hyper-endemic tuberculosis areas.
\end{abstract}

Keywords: Prisons; Tuberculosis, cutaneous; Spain; Diagnosis, Differential; Endemic Diseases; Abscess; HIV. 


\section{INTRODUCCIÓN}

En 2010, hubo casi nueve millones de casos nuevos de tuberculosis (TB), que ocasionaron 350.000 muertes en infectados por el VIH y 1,1 millones de muertes en no infectados por el VIH. A pesar de estos datos, el número absoluto de casos de TB ha ido disminuyendo desde 2006, en vez de aumentar lentamente como se preveía que ocurriría ${ }^{1}$. La forma de presentación clásica de la TB es la pulmonar, aunque los bacilos tuberculosos diseminados durante la infección inicial pueden alojarse en estado latente en todos los tejidos. Las formas extrapulmonares suponen el $10-20 \%$ de los casos de TB en enfermos inmunocompetentes, pero este tipo de presentación se incrementa mucho en las personas con inmunodeficiencia. Las localizaciones extrapulmonares más frecuentes son los ganglios linfáticos, el sistema osteoarticular, el aparato génito-urinario, las meninges y el encéfalo. Otras presentaciones, como las cutáneas, representan menos del $0,5 \%$ de los casos en los países industrializados y su frecuencia es todavía menor si excluimos las becegeitis (TB debidas a vacunación por BCG) o las tuberculides. El objetivo de este trabajo es presentar el caso de un joven, sin inmunodeficiencia congénita o adquirida, en el que se diagnosticó una TB diseminada a partir de un absceso de partes blandas localizado a nivel lumbar.

\section{MÉTODOS}

Caso clínico estudiado y diagnosticado entre marzo y mayo de 2012 por los servicios médicos del centro penitenciario de Hombres de Barcelona (CPHB) y por el Servicio de Medicina Interna del Hospital Consorci Sanitari de Terrassa (HCST). Para la descripción, se recogen los datos de la historia clínica del paciente, de sus informes de atención hospitalaria (de urgencia y de estancia programada) y de las pruebas complementarias practicadas.

\section{RESULTADOS}

Varón de 35 años, natural de Cabo Verde, en España desde los 4 años de edad. Actualmente, preso en Barcelona. Fumador de 10 cigarros/día hasta hace 5 meses y consumidor ocasional de marihuana. No otros hábitos tóxicos. Antecedente de múltiples fracturas, algunas precisaron cirugía, tras precipitación desde un cuarto piso hace 16 meses. En 2008, intradermorreacción de Mantoux (IDRM) de $25 \mathrm{~mm}$. Hace aproximadamente un mes y medio, inició cuadro de dolor lumbar, sensación distérmica, escalofríos, disuria, así como calambres y parestesias. Recibió tratamiento con antiinflamatorios y norfloxacino, sin mejoría. Por ese motivo, fue trasladado de urgencias a centro hospitalario. La analítica mostró PCR de 16,27 $\mathrm{mg} / \mathrm{dl}$, leucocitos normales con neutrofilia y linfopenia relativas, anemia normocítica leve, trombocitosis $(448000 / \mathrm{mcl})$, TP $65 \%$ y fibrinógeno $9 \mathrm{~g} / \mathrm{l}$. El estudio radiológico abdominal y de columna lumbar no mostró hallazgos patológicos. Se solicitó ecografía renovesico-prostática, que descartó dilatación de la vía excretora por obstrucción. No litiasis. Se detectó un pequeño nódulo de $1,2 \mathrm{~cm}$ en lóbulo medio prostático, inespecífico, a controlar evolutivamente. Se solicitó urinocultivo y con orientación de posible infección de vías urinarias altas retornó al centro penitenciario con tratamiento de cefixima $400 \mathrm{mg} / 24 \mathrm{~h}$ durante 3 semanas, a la espera de resultados del urinocultivo y ajuste posterior en función del antibiograma.

Días después, se comunicó telefónicamente resultado negativo del urinocultivo y se realizó en prisión una ecografia que mostró mínima cantidad de derrame pleural derecho. Dada la persistencia de la sintomatología y la aparición de síndrome constitucional (pérdida de peso, astenia, anorexia y fiebre termometrada de $37,5-38^{\circ}$, preferentemente vespertina) se tramitó ingreso programado para estudio en el HCST.

$\mathrm{Al}$ ingreso, el paciente se encontraba febril $\left(39^{\circ} \mathrm{C}\right)$ con exploración anodina a excepción de palpación de masa, probablemente de partes blandas, de $5 \mathrm{~cm}$ de diámetro en región lumbar (figura 1). Los marcadores tumorales, las serologias (lues, hepatitis B y C, VIH) y los hemocultivos fueron negativos. El sedimento de orina, normal. La radiografia de tórax mostró derrame pleural derecho con loculación-engrosamiento pleural a nivel superoanterior y ensanchamiento mediastínico medio con ocupación paratraqueal baja, subcarinal y parahiliar derecha. También, masa (4 cm aproximadamente) mal delimitada en LSD con contacto pleural y pinzamiento del seno costofrénico derecho. El TAC toraco-abdominal mostró en parénquima pulmonar un nódulo apical derecho cavitado $(1,8 \times 1,6 \mathrm{~cm})$ con micronódulos adyacentes, un nódulo de $6 \mathrm{~mm}$ en LSD y atelectasia subsegmentaria en LM, engrosamiento nodular de la pleura apical derecha y afectación de la pleura mediastínica con importante derrame pleural izquierdo. Asimismo, colecciones hipodensas en musculatura intercostal, músculo aductor derecho y en musculatura paravertebral derecha $(5,8 \times 3 \times 2,5 \mathrm{~cm})$ e izquierda $(4,4 \times 3,2 \times 1,6$ y $1,9 \times 1,5 \mathrm{~cm})$. Se realizó toracocentesis en hemitórax izquierdo que obtuvo $40 \mathrm{cc}$ de líquido amarillento (ADA elevado -2,2 ukat/L,- y 


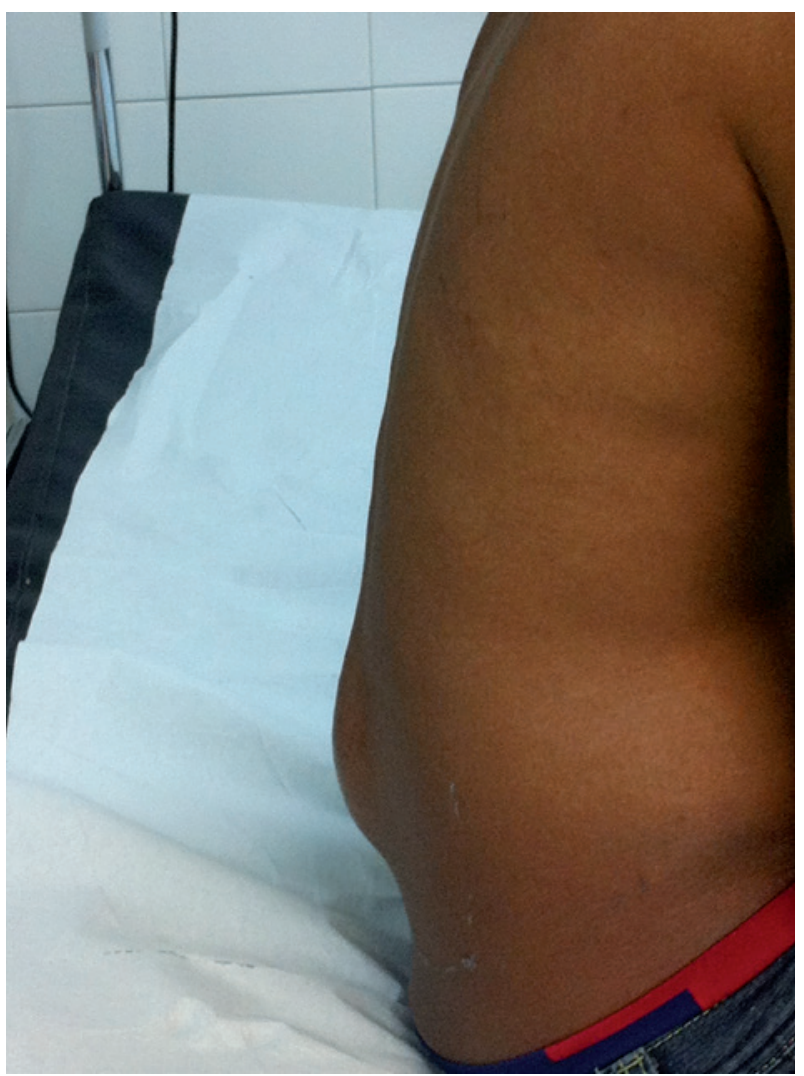

Figura 1. Absceso de partes blandas situado a nivel lumbar. Imagen lateral.

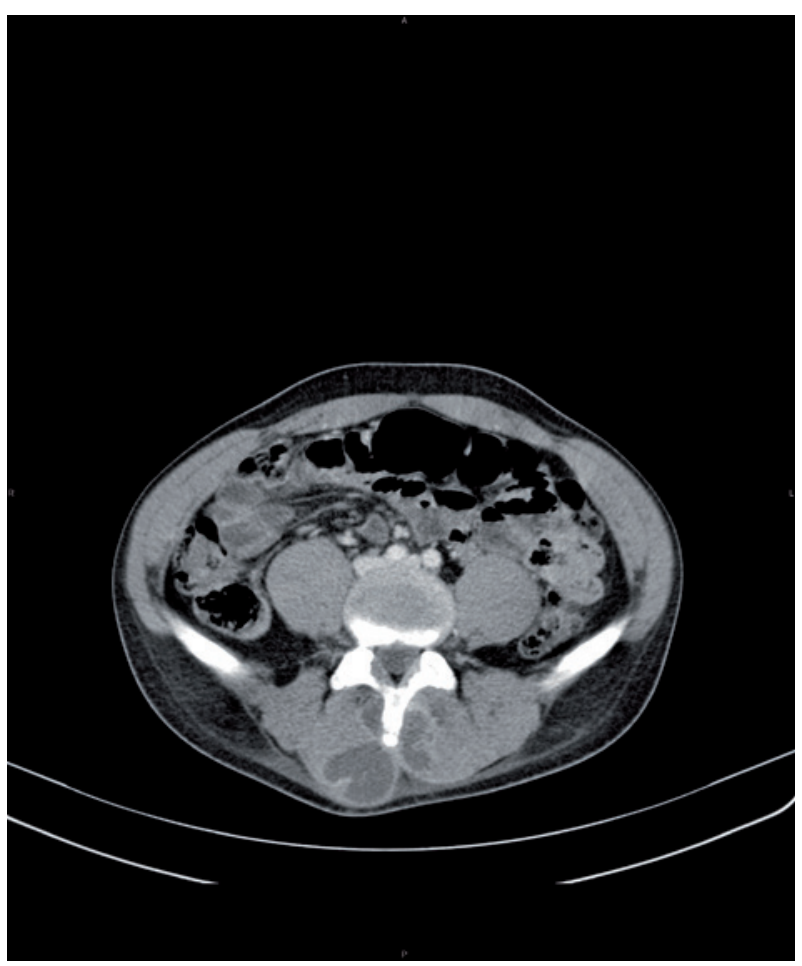

Figura 2. Colecciones en la musculatura paravertebral lumbar, de localización bilateral, en la Tomografía Computarizada (TC). citología negativa para células malignas). La punción aspirativa de la tumoración lumbar izquierda permitió la salida de $10 \mathrm{cc}$ de líquido purulento que fue positivo para $M$. tuberculosis mediante determinación de PCR y cultivo en medio de Lowenstein. Se solicitó subpoblaciones linfocitarias (CD3, CD4, CD8 y cocientes) con resultados dentro de la normalidad. El antibiograma mostró sensibilidad a todos los fármacos antituberculosos testados. No se pudieron realizar baciloscopias de esputo por nula expectoración. Se inició tratamiento antituberculoso con cuatro drogas y aislamiento respiratorio por la lesión cavitada pulmonar. Tras desaparición de la fiebre, del dolor de la masa lumbar, mejoría importante del estado general y disminución del tamaño del derrame pleural izquierdo, el paciente fue dado de alta con diagnóstico de TB diseminada (pleuro-pulmonar y en abscesos musculares). De acuerdo con el servicio de Cirugía, se manejó la tumoración lumbar (crecimiento con signos de fluctuación) con punciones aspirativas de repetición (figura 2).

\section{DISCUSIÓN}

Los abscesos tuberculosos son complicaciones relativamente comunes de la espondilodiscitis tuberculosa ${ }^{2,3}$ y no es infrecuente que, en estos casos, haya afectación también del psoas o de los músculos paravertebrales, incluso años más tarde del diagnóstico de la TB${ }^{4}$. Aunque no es habitual, también se han descrito casos de abscesos tuberculosos, meses después de la vacunación con $\mathrm{BCG}^{5}$. Los abscesos fríos o gomas sin embargo, son producto de la diseminación metastásica, vía hematógena, de micobacterias latentes, formando abscesos en las extremidades o el tronco, generalmente sin compromiso de los tejidos profundos. Este es, probablemente, el proceso fisiopatogénico que ocurrió en este paciente. Hay pocos casos de goma tuberculosa descritos en la literatura $\mathrm{y}$, aunque son más habituales en pacientes de países con alta endemia de $\mathrm{TB}^{6,7}$ también se han descrito en países de baja endemia como Japón ${ }^{8}$ o EE.UU ${ }^{9}$. No obstante, en los últimos años, ha habido un aumento en la prevalencia de estas presentaciones, asociada a enfermedades con inmunodepresión ${ }^{10}$, entre ellas la infección por el VIH ${ }^{11}$. También se ha sugerido que este tipo de presentaciones pueden ser más frecuentes en reclusos ${ }^{12}$, sobre todo si pertenecen a prisiones - como en nuestro caso - en las que más del $61 \%$ de los ingresados actualmente son inmigrantes, muchos de países con alta endemia de TB, y donde la prevalencia de esta infección supera el $40 \%{ }^{13}$. 
Debe recordarse, finalmente, que el caso que nos ocupa llegó a España a los 4 años de edad. Pudo infectarse en Cabo Verde - donde la tasa de incidencia de TB es de 147 casos por 100.000 , cinco veces superior a España- o luego en nuestro país y es también muy probable que estuviera vacunado por $\mathrm{BCG}^{14}$. Un aspecto a comentar es que en 2008 no se prescribió tratamiento para la infección tuberculosa latente, y aunque no era una indicación absoluta ${ }^{15}$, el tamaño de la IDRM y la estancia en prisión son factores que aconsejan indicarla tras descartar el diagnóstico de TB.

En definitiva, el caso presentado sugiere que debe mantenerse una vigilancia activa sobre lesiones que aunque infrecuentes pueden ser secundarias a infección por $M$ tuberculosis, especialmente en población inmunocomprometida y en inmigrantes procedentes de zonas hiperendémicas de TB .

\section{CORRESPONDENCIA:}

Dr. Andrés Marco

Servicios Médicos del Centro Penitenciario de

Hombres de Barcelona.

C/Entenza 155. Barcelona 08029.

andres.marco.m@gmail.com

\section{REFERENCIAS BIBLIOGRÁFICAS}

1. World Health Organization. Global Tuberculosis Control. Who Report 2011 Geneva: WHO; 2011 [citado 2012 May 29]. Disponible en: Http://www.who.int/tb/publications/global_report/2011/es/index.html

2. Lobato Z, Artigas S, Séculi JL. Abscesos cutáneos como forma de presentación de la enfermedad de Pott. An Pediatr (Barc). 2004; 61: 66-8.

3. Abu-Salem OT. Tuberculous abscesses of the quadriceps femoris muscle without osseous involvement. East Mediterr Health J. 2000; 6: 1136-8.

4. Korovessis P, Papadaki E, Repanti M, Stamatakis M. Latent solitary tuberculous psoas abscess 52 years after healed thoracolumbar tuberculous spondylitis. Spine (Phila Pa 1976). 1995; 20: 1709-12.
5. Rajapakse CD, Shingadia D. Tuberculous pyomyositis of the left quadratus lumborum. Arch Dis Child. 2006; 91: 512.

6. Sen RK, Triapathy SK, Deivaraju C, Das A. Isolated focal pyomyositis of teres minor: an unusual presentation of tuberculosis. Acta Orthop Traumatol Turc. 2011; 45: 276-9.

7. Sabat D, Kumar V. Primary tuberculous abscess of rectus femoris muscle: a case report. J Infect Dev Ctries. 2009; 3: 476-8.

8. Saika A, Kanno T, Ishiguchi T, Oka H, Shiotani A, Yamanishi T, et al. A case of the tuberculous abscess in the abdominal wall. Kansenshogaku Zasshi. 1997; 71: 540-4

9. Huang DY. Tuberculous muscle abscess: an unusual presentation of tuberculosis. Am J Med. 1990; 88: 57N-59N.

10. Ergin F, Arslan H, Bilezikçi B, Ağildere AM, Ozdemir N. Primary tuberculosis in the gluteal muscle of a patient with chronic renal failure. A rare presentation. Nephron. 2001; 89: 463-6.

11. Lupatkin H, Bräu N, Flomenberg P, Simberkoff MS. Tuberculous abscesses in patients with AIDS. Clin Infect Dis. 1992; 14: 1040-4.

12. Pérez B, Pifarre R, de Vera CV, García M, Baradad M, Vilà M, et al. Tuberculosis cutánea por Mycobacterium tuberculosis, una patología muy poco frecuente. An Med Interna (Madrid). 2006; 23: 560-1.

13. Marco A, Solé N, Orcau A, Escribano M, del Baño L, Quintero S, et al. Prevalence of latent tuberculosis infection in inmates recently admitted to a men's prison in Barcelona. Int J Tuberc Lung Dis. 2012; 16: 60-4.

14. El Banco Mundial [Internet]. Whasington: Banco Mundial; 2014 [actualizado 2014; citado 2012 May 25]. Incidencia de tuberculosis (por cada 100.000 personas); [sobre 2 pantallas] Disponible en: http://datos.bancomundial.org/indicador/ SH.TBS.INCD/countries/

15. Ruiz F, López G y Grupo de Trabajo del Documento. Documento de Consenso para el control de la tuberculosis en las prisiones españolas (resumen). Rev Esp Sanid Penit 2010; 12: 64-78. 